\title{
Conjunctive Queries for a Tractable Fragment of OWL $1.1^{\star}$
}

\author{
Markus Krötzsch, Sebastian Rudolph, and Pascal Hitzler \\ Institut AIFB, Universität Karlsruhe, Germany
}

\begin{abstract}
Despite the success of the Web Ontology Language OWL, the development of expressive means for querying OWL knowledge bases is still an open issue. In this paper, we investigate how a very natural and desirable form of queries-namely conjunctive ones - can be used in conjunction with OWL such that one of the major design criteria of the latter — namely decidability—can be retained. More precisely, we show that querying the tractable fragment $\mathcal{E} \mathcal{L}^{++}$ of OWL 1.1 is decidable. We also provide a complexity analysis and show that querying unrestricted $\mathcal{E} \mathcal{L}^{++}$is undecidable.
\end{abstract}

\section{Introduction}

Conjunctive queries originated from research in relational databases [1], and, more recently, have also been identified as a desirable form of querying expressive description logics (DLs) that are underlying OWL [2 3 4 56]. At the same time, tractable fragments of OWL are receiving increasing attention as they promise to provide a favourable balance between expressivity and scalability. Such fragments have, in particular, been identified as part of the OWL 1.1 proposal 1 and this raises the question how conjunctive queries can be combined favourably with the underlying description logics.

In this paper, we thus present the very first algorithm for answering conjunctive queries in the tractable $\mathcal{E} \mathcal{L}^{++}$-fragment of $\mathcal{S R O I Q ~ [ 7 8 ] , ~ a n d ~ t h u s ~ o f ~ O W L ~ 1 . 1 . ~ T h e ~}$ algorithm is based on an automata-theoretic formulation of complex role inclusion axioms that was also found useful in reasoning with SROIQ [910].

Our algorithm in particular allows us to derive a number of complexity results related to conjunctive query answering in $\mathcal{E} \mathcal{L}^{++}$. We first show that conjunctive queries in $\mathcal{E} \mathcal{L}^{++}$are undecidable in general, and identify the $\mathcal{E} \mathcal{L}^{++}$-fragment of $\mathcal{S R O I Q}$ as an appropriate decidable sub-DL. Under some related restrictions of role inclusion axioms, we show that conjunctive query answering in general is PSPACE-complete. Query answering for fixed knowledge bases (query complexity) is shown to be NP-complete, whereas for fixed queries (schema complexity) it is merely P-complete.

\section{Preliminaries}

We assume the reader to be familiar with the basic notions of description logics (DLs). The DLs that we will encounter in this paper are $\mathcal{E} \mathcal{L}^{++}[7$ and, marginally, $\mathcal{S} R O I Q$

\footnotetext{
* This work has been supported by the European Commission under contract IST-2006-027595 $\mathrm{NeOn}$, and by the Deutsche Forschungsgemeinschaft (DFG) under the ReaSem project.

${ }^{1}$ See http://webont. org/owl/1.1/for both.
} 
[10]. A DL signature consists of a finite set of role names $\mathbf{R}$, a finite set of individual names $\mathbf{I}$, and a finite set of concept names $\mathbf{C}$. We will use this notation throughout the paper. $\mathcal{E} \mathcal{L}^{++}$supports nominals, which we conveniently represent as follows: for any $a \in \mathbf{I}$, there is a concept $\{a\} \in \mathbf{C}$ such that $\{a\}^{\mathcal{I}}=\left\{a^{I}\right\}$ (for any interpretation $\mathcal{I}$ ). As shown in [7], any $\mathcal{E} \mathcal{L}^{++}$knowledge base is equivalent to one in normal form, only containing the following axioms:
TBox: $A \sqsubseteq C$
$A \sqcap B \sqsubseteq C$
RBox: $R \sqsubseteq T$
$R \circ S \sqsubseteq T$
$A \sqsubseteq \exists R . C$
$\exists R . A \sqsubseteq C$

where $A, B \in \mathbf{C} \cup\{\top\}, C \in \mathbf{C} \cup\{\perp\}$, and $R, S, T \in \mathbf{R}$. Note that ABox statements of the forms $C(a)$ and $R(a, b)$ are internalised into the TBox. The standard model theoretic semantics of $\mathcal{E} \mathcal{L}^{++}$can be found in [7]. Unless otherwise specified, the letters $C, D, E$ in the remainder of this work always denote (arbitrary) concept names, and the letters $R, S$ denote (arbitrary) role names. We do not consider concrete domains in this paper, but are confident that our results can be extended accordingly.

For conjunctive queries, we largely adopt the notation of [6] but directly allow for individuals in queries. Let $\mathbf{V}$ be a countable set of variable names. Given elements $x$, $y \in \mathbf{V} \cup \mathbf{I}$, a concept atom (role atom) is an expression $C(x)$ with $C \in \mathbf{C}(R(x, y)$ with $R \in \mathbf{R})$. A conjunctive query $q$ is a set of concept and role atoms, read as a conjunction of its elements. By $\operatorname{Var}(q)$ we denote the set of variables occurring in $q$. Consider an interpretation $\mathcal{I}$ with domain $\Delta^{I}$, and a function $\pi: \operatorname{Var}(q) \cup \mathbf{I} \rightarrow \Delta^{\mathcal{I}}$ such that $\pi(a)=a^{I}$ for all $a \in \mathbf{I}$. We define

$$
\mathcal{I}, \pi \mid=C(x) \text { if } \pi(x) \in C^{\mathcal{I}}, \quad \text { and } \quad \mathcal{I}, \pi \models R(x, y) \text { if }(\pi(x), \pi(y)) \in R^{\mathcal{I}} .
$$

If there is some $\pi$ such that $\mathcal{I}, \pi \models A$ for all atoms $A \in q$, we write $\mathcal{I} \models q$ and say that $\mathcal{I}$ entails $q$. We say that $q$ is entailed by a knowledge base $K B$, denoted $K B \models q$, if all models of $K B$ entail $q$.

We conclude this section with an important result on conjunctive queries in $\mathcal{E} \mathcal{L}^{++}$.

Theorem 1. For an $\mathcal{E} \mathcal{L}^{++}$knowledge base $K B$ and a conjunctive query $q$, the entailment problem $K B \models q$ is undecidable. Likewise, checking class subsumptions in $\mathcal{E} \mathcal{L}^{++}$ extended with inverse roles or role conjunctions is undecidable, even if those operators occur only in the concepts whose subsumption is checked.

Intuitively, the result holds since RBoxes can encode context-free languages, the intersection of which can then be checked with conjunctive queries/inverse roles/role conjunctions. This problem is undecidable (see [11] for a proof). Clearly, arbitrary role compositions are too expressive when aiming for a decidable (or even tractable) logic that admits conjunctive queries. We thus restrict our attention to the fragment of $\mathcal{E} \mathcal{L}^{++}$ that is in the (decidable) description logic SROIQ [10], and investigate its complexity with respect to conjunctive query answering.

Definition 1. An $\mathcal{E} \mathcal{L}^{++}$RBox in normal form is regular if there is a strict partial order $<$ on $\mathbf{R}$ such that, for all role inclusion axioms $R_{1} \sqsubseteq S$ and $R_{1} \circ R_{2} \sqsubseteq S$, we find $R_{i}<S$ or $R_{i}=S(i=1,2)$. An $\mathcal{E} \mathcal{L}^{++}$knowledge base is regular if it has a regular $R B o x$.

The existence of $\prec$ ensures that the role hierarchy does not contain cyclic dependencies other than through direct recursion of a single role. 
Table 1. Closure rules for an interpretation $\mathcal{I}$ w.r.t. some knowledge base $K B$. In general, we assume that $C, D \in \mathbf{C} \cup\{\mathrm{T}, \perp\}$ and $R_{1}, R_{2}, S \in \mathbf{R}$.
(1) $\frac{\delta \in C^{I} \quad K B \models C \sqsubseteq D}{D^{I}:=D^{I} \cup\{\delta\}}$
(2) $\frac{\delta \in C^{I} \quad K B \models C \sqsubseteq \exists R . D \quad K B \not \models D \sqsubseteq\{a\} \text { for any } a \in \mathbf{I}}{\Delta_{I}:=\Delta_{I} \cup\{\epsilon\} \quad R^{I}:=R^{I} \cup\{(\delta, \epsilon)\} \quad D^{I}:=D^{I} \cup\{\epsilon\}}$ where $\epsilon=\epsilon_{\delta, C \sqsubseteq \exists R . D}$
(3) $\frac{\delta \in C^{I} \quad K B \vDash C \sqsubseteq \exists R \cdot D \quad K B \models D \sqsubseteq\{a\} \text { for some } a \in \mathbf{I}}{R^{I}:=R^{I} \cup\{(\delta, a)\}}$
(4) $\frac{(\delta, \epsilon) \in R^{I} \quad R \sqsubseteq S \in K B}{S^{I}:=S^{I} \cup\{(\delta, \epsilon)\}}$
(5) $\frac{(\delta, \epsilon) \in R_{1}^{I} \quad(\epsilon, \gamma) \in R_{2}^{I} \quad R_{1} \circ R_{2} \sqsubseteq S \in K B}{S^{I}:=S^{I} \cup\{(\delta, \gamma)\}}$

\section{Canonical Models and Reasoning Automata for $\mathcal{E} \mathcal{L}^{++}$}

$\mathcal{E} \mathcal{L}^{++}$, like all Horn-DLs, allows for the construction of canonical or universal models. By this we mean an interpretation that is in a sense most general among the models of a given $\mathcal{E} \mathcal{L}^{++}$knowledge base, satisfying exactly those formulae that are logical consequences of the knowledge base. This notion could be formalised further (using the concept of (bi)simulation between models), but we merely require canonical models to guide us in the development and verification of a query answering algorithm, and hence we will confine ourselves to directly showing the relevant properties.

Consider a regular consistent $\mathcal{E} \mathcal{L}^{++}$knowledge base $K B$. Here and in the following, we assume w.l.o.g. that $K B$ does not entail $a \approx b$ (i.e. $\{a\} \equiv\{b\}$ ) for any $a, b \in \mathbf{I}$. Indeed, one can just replace all occurrences of $b$ with $a$ in this case, both within $K B$ and within any query we wish to consider later on (and this case can be detected in polynomial time). Moreover, we assume that there is at least one individual in the language, i.e. I $\neq \emptyset$. We now provide an iterative construction of a model $\mathcal{I}$ of $K B$. Our goal is to obtain a concise definition of a suitable canonical model, so it is no matter of concern that the given construction does not terminate after finitely many steps.

To simplify our arguments, we adopt a naming scheme for potential elements of the domain of $\mathcal{I}$. Let $\Delta$ be the smallest set such that $\mathbf{I} \subseteq \Delta$ and, for any $\delta \in \Delta, C, D \in \mathbf{C}$, and $R \in \mathbf{R}$, we find that $\epsilon_{\delta, C \sqsubseteq \exists R . D} \in \Delta$. We will define $\mathcal{I}$ such that $\Delta_{\mathcal{I}} \subseteq \Delta$.

For any two interpretations $\mathcal{J}_{1}$ and $\mathcal{J}_{2}$ of $K B$, we say that $\mathcal{J}_{1}$ is smaller than (or equal to) $\mathcal{J}_{2}$ if, for any $F \in \mathbf{C} \cup \mathbf{R} \cup\{\top\}, F^{\mathcal{J}_{1}} \subseteq F^{\mathcal{J}_{2}}$. The interpretation $\mathcal{I}$ is defined to be the smallest interpretation such that $\Delta_{\mathcal{I}} \subseteq \Delta,\{a\}^{\mathcal{I}}:=a$ for all $a \in \mathbf{I}$, and $\mathcal{I}$ is closed under the rules of Table 1 It is easy to see that this smallest interpretation exists.

The rules of Table 1 have the special property that each individual is "initialised" with at most one concept name. Formally, we define for each element $\delta \in \Delta_{I}$ a concept name $\iota(\delta)$ as follows:

- if $\delta \in \mathbf{I}, \iota(\delta):=\{\delta\}$,

- if $\delta=\epsilon_{\delta^{\prime}}, C \sqsubseteq \exists R . D$ for some $\delta^{\prime} \in \Lambda_{I}, C, D \in \mathbf{C}, R \in \mathbf{R}$, then $\iota(\delta):=D$.

Note that the above cases are indeed exhaustive and mutually exclusive. 


\section{Lemma 1. The interpretation I as constructed above is a model of $K B$.}

Proof. First note that the domain of $\mathcal{I}$ is non-empty since we assume the existence of at least one individual. We have to check that all axioms of $K B$ are indeed satisfied. For axioms of the form $C \sqsubseteq \exists R$.D this is obvious by rules (2) and (3) of Table 1 . Similarly, all role inclusion axioms are directly accounted for by rules (4) and (5).

So it remains to show that axioms $\Phi$ of the forms $C \sqsubseteq D, \exists R . C \sqsubseteq D$, and $C_{1} \sqcap C_{2} \sqsubseteq D$ are satisfied. Obviously, whenever $\delta \in C^{I}\left(\delta \in \exists R\right.$. $\left.C^{I}\right)$ for some $C \in \mathbf{C}$ (and $R \in \mathbf{R}$ ), we find $K B \models \iota(\delta) \sqsubseteq C(K B \models \iota(\delta) \sqsubseteq \exists R . C)$. We conclude that, whenever the premise of some axiom $\Phi$ as above is satisfied for $\delta$, then it is entailed by $\iota(\delta)$, and so its conclusion $D$ is a direct consequence of $\iota(\delta)$ under $K B$. Thus $\Phi$ is satisfied by rule (1).

We are most interested in the specific structure of the canonical model. Its construction attempts to preserve a form of tree-likeness, broken only by the potential occurrence of nominals. Formally, this is expressed through the following property.

Property 1. For any element $\delta \in \Delta_{I}$ that is not an individual $(\delta \notin \mathbf{I})$, there is a unique chain of elements $\delta_{0} \ldots \delta_{k}=\delta$ and role names $R_{0}, \ldots, R_{k-1} \in \mathbf{R}$, such that $\delta_{0} \in \mathbf{I}$ and, for all $i=1, \ldots, k, \delta_{i} \in \Delta_{I}$ is of the form $\delta_{\epsilon, C \sqsubseteq R . D}$ with $\epsilon=\delta_{i-1}$ and $R=R_{i-1}$. This is easily verified by observing that any $\delta$ of the given form must have been entailed by rule (2), and by applying a simple induction on the depth of this entailment. In this case, we say that $\delta_{i}$ generates $\delta$ via the roles $R_{i} \ldots R_{k}(i=0, \ldots, k)$.

The canonicity of the model $\mathcal{I}$ manifests itself in the fact that structures in the model are necessary logical consequences of given axioms.

Property 2. Consider elements $\delta, \epsilon \in \Delta_{I}$ such that $\delta$ generates $\epsilon$ via the roles $R_{0} \ldots R_{k}$. Then $\iota(\delta) \sqsubseteq \exists R_{0} .\left(\ldots \exists R_{k} . \iota(\epsilon) \ldots\right)$ is a consequence of $K B$. This is obvious by another simple inductive argument that utilises the preconditions of the applications of rule (3).

Property 3. For any $(\delta, \epsilon) \in R^{I}$, there is a chain of elements $\delta=\delta_{0} \ldots \delta_{k}=\epsilon$ and role names $R_{i}(i=0, \ldots, k-1)$, such that

- $\left(\delta_{i}, \delta_{i+1}\right) \in R_{i}^{I}$ is directly entailed by one of rules (2) and (3), and

- $R_{0} \circ \ldots \circ R_{k-1} \sqsubseteq R$ is a consequence of $K B$.

We show this by an inductive argument as follows: for the base case, assume that $(\delta, \epsilon) \in R^{I}$ follows from rule (2) or (3). Then the above condition clearly holds. For the induction step, assume that $(\delta, \epsilon) \in R^{I}$ follows by applying rule (5) to $R_{1} \circ R_{2} \sqsubseteq R$, and that the claim holds for the statements $\left(\delta, \delta_{j}\right) \in R_{1}^{I}$ and $\left(\delta_{j}, \epsilon\right) \in R_{2}^{I}$. We easily can construct from these assumptions a suitable chain of elements from the chains postulated for $R_{1}$ and $R_{2}$. Similarly, the second condition of the claim follows from the assumption that $R_{1} \circ R_{2} \sqsubseteq R$ and the induction hypothesis. Rule (4) is treated analogously.

In the remainder of this section, we investigate various means of presenting logical inferences by means of automata. These encodings will play a major role within our subsequent query answering algorithm. We describe nondeterministic finite automata (NFA) $\mathcal{A}$ as tuples $\left(Q_{\mathcal{A}}, \Sigma_{\mathcal{A}}, \delta_{\mathcal{H}}, i_{\mathcal{A}}, F_{\mathcal{A}}\right)$, where $Q_{\mathcal{A}}$ is a finite set of states, $\Sigma_{\mathcal{A}}$ is a finite alphabet, $\delta_{\mathcal{A}}: Q_{\mathcal{H}} \times Q_{\mathcal{A}} \rightarrow 2^{\Sigma_{\mathcal{P}}}$ is a transition function that maps pairs of states to sets of alphabet symbols $\sqrt[2]{-2} i_{\mathcal{A}}$ is the initial state, and $F_{\mathcal{A}}$ is a set of final states.

\footnotetext{
${ }^{2}$ A possibly more common definition is to map pairs of states and symbols to sets of states, but the above is more convenient for our purposes.
} 
Table 2. Completion rules for constructing an NFA from an $\mathcal{E} \mathcal{L}^{++}$knowledge base $K B$

(CR1) If $C^{\prime} \in \delta(C, C), C^{\prime} \sqsubseteq D \in K B$, and $D \notin \delta(C, C)$ then $\delta(C, C):=\delta(C, C) \cup\{D\}$.

(CR2) If $C_{1}, C_{2} \in \delta(C, C), C_{1} \sqcap C_{2} \sqsubseteq D \in K B$, and $D \notin \delta(C, C)$ then $\delta(C, C):=\delta(C, C) \cup\{D\}$.

(CR3) If $C^{\prime} \in \delta(C, C), C^{\prime} \sqsubseteq \exists R . D \in K B$, and $R \notin \delta(C, D)$ then $\delta(C, D):=\delta(C, D) \cup\{R\}$.

(CR4) If $R \in \delta(C, D), D^{\prime} \in \delta(D, D), \exists R . D^{\prime} \sqsubseteq E \in K B$, and $E \notin \delta(C, C)$ then $\delta(C, C):=$ $\delta(C, C) \cup\{E\}$.

(CR5) If $R \in \delta(C, D), \perp \in \delta(D, D)$, and $\perp \notin \delta(C, C)$ then $\delta(C, C):=\delta(C, C) \cup\{\perp\}$.

(CR6) If $\{a\} \in \delta(C, C) \cap \delta(D, D)$, and there are states $C_{1}, \ldots, C_{n}$ such that

- $C_{1} \in\{C, \top, A\} \cup\{\{b\} \mid b \in \mathbf{I}\}$,

- $\delta\left(C_{j}, C_{j+1}\right) \neq \emptyset$ for all $j=1, \ldots, n-1$,

$-C_{n}=D$,

and $\delta(D, D) \nsubseteq \delta(C, C)$ then $\delta(C, C):=\delta(C, C) \cup \delta(D, D)$.

(CR7) If $R \in \delta(C, D), R \sqsubseteq S$, and $S \notin \delta(C, D)$ then $\delta(C, D):=\delta(C, D) \cup\{S\}$.

(CR8) If $R_{1} \in \delta(C, D), R_{2} \in \delta(D, E), R_{1} \circ R_{2} \sqsubseteq S$, and $S \notin \delta(C, E)$ then $\delta(C, E):=\delta(C, E) \cup\{S\}$.

Proposition 1. Given a regular $\mathcal{E} \mathcal{L}^{++}$RBox, and some role $R \in \mathbf{R}$, there is an NFA $\mathcal{A}(R)$ over the alphabet $\mathbf{R}$ which accepts a word $R_{1} \ldots R_{n}$ iff $R_{1} \circ \ldots \circ R_{n} \sqsubseteq R$ is a consequence of every $\mathcal{E} \mathcal{L}^{++}$knowledge base with the given RBox.

One possible construction for the required automaton is discussed in [10]. Intuitively, the RBox can be understood as a grammar for a regular language, for which an automaton can be constructed in a canonical way. The required construction of $\mathcal{A}(R)$ might be exponential for some RBoxes. In [9], restrictions have been discussed that prevent this blow-up, leading to NFA of only polynomial size w.r.t. the RBox. Accordingly, an RBox is simple whenever, for all axioms of the form $R_{1} \circ S \sqsubseteq S, S \circ R_{2} \sqsubseteq S$, the RBox does not contain a common subrole $R$ of $R_{1}$ and $R_{2}$ for which there is an axiom of the form $R \circ S^{\prime} \sqsubseteq R^{\prime}$ or $S^{\prime} \circ R \sqsubseteq R^{\prime}$. We will usually consider only such simple RBoxes whenever the size of the constructed automata matters.

Next we describe the construction of a novel kind of automaton that encodes certain concept subsumptions entailed by an $\mathcal{E} \mathcal{L}^{++}$knowledge base. The automaton itself is closely related to the reasoning algorithm given in [7], but the representation of entailments via nondeterministic finite automata (NFA) will be essential for the query answering algorithm in the following section.

Consider an $\mathcal{E} \mathcal{L}^{++}$knowledge base $K B$. Given a concept name $A \in \mathbf{C}$, we construct an NFA $\mathcal{A}_{K B}(A)=(Q, \Sigma, \delta, i, F)$ that computes superconcepts of $A$, where we omit the subscript if $K B$ is clear from the context. Set $Q=F=\mathbf{C} \cup\{\top\}, \Sigma=\mathbf{C} \cup \mathbf{R} \cup\{\top, \perp\}$, and $i=A$. The transition function $\delta$ is initially defined as $\delta(C, C):=\{C, \top\}$ (for all $C \in Q$ ) and $\delta(C, D):=\emptyset$ (for all $C, D \in Q$ with $C \neq D$ ), and extended iteratively by applying the rules in Table 2] The rules correspond to completion rules in [7, Table 2], though the conditions for (CR6) are slightly relaxed, fixing a minor glitch in the original algorithm.

It is easy to see that the rules of Table 2 can be applied at most a polynomial number of times. The words accepted by $\mathcal{A}(A)$ are strings of concept and role names. For each such word $w$ we inductively define a concept expression $C_{w}$ as follows: 
- if $w$ is empty, then $C_{w}=\mathrm{T}$,

- if $w=R v$ for some $R \in \mathbf{R}$ and word $v$, then $C_{w}=\exists R .\left(C_{v}\right)$,

- if $w=C v$ for some $C \in \mathbf{C}$ and word $v$, then $C_{w}=C \sqcap C_{v}$.

For instance, the word $C R D E S$ translates into $C_{C R D E S}=C \sqcap \exists R .(D \sqcap E \sqcap \exists S . \top)$. Based on the close correspondence of the above rules to the derivation rules in [7], we can now establish the main correctness result for the automaton $\mathcal{A}(A)$.

Theorem 2. Consider a knowledge base KB, concept $A$, and NFA $\mathcal{A}(A)$ as above, and let $w$ be some word over the associated alphabet. Then $K B \models A \sqsubseteq C_{w}$ iff one of the following holds:

- $\mathcal{A}(A)$ accepts the word $w$, or

- there is a transition $\perp \in \delta(C, C)$ where $C=\mathrm{T}, C=A$, or $C=\{a\}$ for some individual a.

In particular, $\mathcal{A}(A)$ can be used to check all subsumptions between $A$ and some atomic concept $B$.

The second item of the theorem addresses the cases where $A$ is inferred to be empty (i.e. inconsistent) or where the whole knowledge base is inconsistent, from which the subsumption trivially follows. While the above yields an alternative formulation of the $\mathcal{E} \mathcal{L}^{++}$reasoning algorithm presented in [7], it has the advantage that it also encodes all paths within the inferred models. This will be essential for our results in the next section. The following definition will be most convenient for this purpose.

Definition 2. Consider a knowledge base KB, concepts $A, B \in \mathbf{C}$, and the NFA $\mathcal{A}(A)=$ $(Q, \Sigma, \delta, i, F)$. The automaton $\mathcal{A}_{K B}(A, B)$ (or just $\mathcal{A}(A, B)$ ) is defined as $\left(Q, \mathbf{R}, \delta^{\prime}, i, F^{\prime}\right)$ where $F^{\prime}=\emptyset$ if $\perp \in \delta(A, A)$ and $F^{\prime}=\{B\}$ otherwise, and $\delta^{\prime}$ is the restriction of $\delta$ to $\mathbf{R}$.

The automaton $\mathcal{A}(A, B)$ normally accepts all words of roles $R_{1}, \ldots, R_{n}$ such that $A \sqsubseteq$ $\exists R_{1}\left(\ldots \exists R_{n} . B \ldots\right)$ is a consequence of $K B$, with the border case where $n=0$ and $K B=$ $A \sqsubseteq B$. Moreover, the language accepted by the NFA is empty whenever $A \sqsubseteq \perp$ has been inferred.

\section{Deciding Conjunctive Queries for $\mathcal{E} \mathcal{L}$}

In this section, we present a nondeterministic algorithm that decides the entailment of a query $q$ with respect to some regular consistent knowledge base $K B$. The algorithm constructs a so-called proof graph which establishes, for all interpretations $\mathcal{I}$ of $K B$, the existence of a suitable function $\pi$ that shows query entailment. Intuitively, a proof graph encodes a fragment of the canonical model $\mathcal{I}$ of Section 3

Formally, a proof graph is a tuple $(N, L, E)$ consisting of a set of nodes $N$, a labelling function $L: N \rightarrow \mathbf{C} \cup\{T\}$, and a partial transition function $E: N \times N \rightarrow \mathbf{A}$, where $\mathbf{A}$ is the set of all NFA over the alphabet $\mathbf{C} \cup\{\top, \perp\} \cup \mathbf{R}$. A node $m \in N$ is reachable if there is some node $n \in N$ such that $E(n, m)$ is defined, and unreachable otherwise. The nodes of the proof graph are abstract representations of elements in the domain of the 
Table 3. A nondeterministic algorithm for deciding conjunctive queries in $\mathcal{E} \mathcal{L}^{++}$

\begin{tabular}{|c|c|}
\hline $\begin{array}{l}\text { A. Query } \\
\text { factorisation }\end{array}$ & $\begin{array}{ll}1 & \text { Select a (possibly empty) set } X \subseteq \operatorname{Var}(q) \\
2 & \text { For each } x \in X \\
3 & \text { Select some } e \in \operatorname{Var}(q) \cup \mathbf{I} \text { and replace all occurrences of } x \text { in } q \text { with } e\end{array}$ \\
\hline $\begin{array}{l}\text { B. Initialise proof } \\
\text { graph }(N, L, E)\end{array}$ & $\begin{array}{rl}4 & N:=\mathbf{I} \cup \operatorname{Var}(q), \text { let } E \text { be undefined for all arguments } \\
5 & \text { For each } a \in \mathbf{I}, L(a):=\{a\} \\
6 & \text { For each } x \in \operatorname{Var}(q) \text {, select } L(x) \in \mathbf{C} \cup\{\mathrm{T}\} \\
7 & \text { For each } n \in N, a \in \mathbf{I}, E(n, a):=\mathcal{A}(L(n), L(a)) \\
8 & \text { While there is an unreachable node } \\
9 & \text { Select some unreachable } x \in \operatorname{Var}(q), \text { select some reachable } n \in N \\
10 & E(n, x):=\mathcal{A}(L(n), L(x))\end{array}$ \\
\hline $\begin{array}{l}\text { C. Check proof } \\
\text { graph }\end{array}$ & $\begin{array}{l}11 \text { For each } n \in N, m \in \operatorname{Var}(q) \\
12 \quad \text { If } E(n, m) \text { is defined and accepts no word, terminate with failure }\end{array}$ \\
\hline $\begin{array}{l}\text { D. Check concept } \\
\text { entailment }\end{array}$ & \begin{tabular}{|l}
13 \\
$14 \quad$ For each concept atom $C(n) \in q$ \\
If not $K B \models L(n) \sqsubseteq C$, terminate with failure
\end{tabular} \\
\hline $\begin{array}{r}\text { E. Split role } \\
\text { automata }\end{array}$ & $\begin{array}{ll}15 & \text { For each role atom } R(n, m) \in q \\
16 & \text { Compute shortest path } n=n_{0}, \ldots, n_{k}=m \text { from } n \text { to } m \\
17 & \text { Split } \mathcal{A}(R) \text { into } k \text { automata } \mathcal{A}\left(R(n, m), n_{0}, n_{1}\right), \ldots, \mathcal{A}\left(R(n, m), n_{k-1}, n_{k}\right) \\
18 & \text { For each } \mathcal{A}\left(R(n, m), n_{i-1}, n_{i}\right) \\
19 & \text { If } \mathcal{A}\left(R(n, m), n_{i-1}, n_{i}\right) \text { accepts no word, terminate with failure }\end{array}$ \\
\hline $\begin{array}{l}\text { F. Check role } \\
\text { entailment }\end{array}$ & 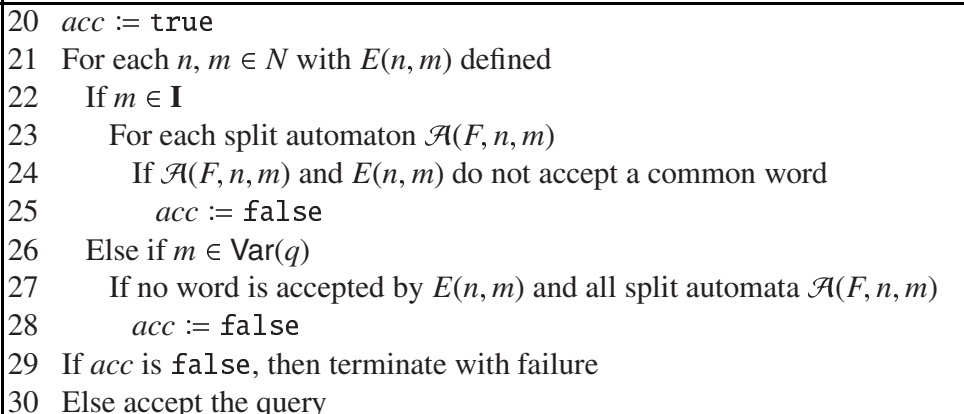 \\
\hline
\end{tabular}

canonical model $\mathcal{I}$ of $K B$. The labels assign a concept to each node, the intuition being that this is the "main concept" $\iota(\delta)$ defined in Section 3 Finally, the transition function encodes role paths in the canonical model, which provide the basis for inferencing about relationships between elements. It would be possible to adopt a more concrete representation for role paths (e.g. by guessing a single path), but our formulation reduces nondeterminism and eventually simplifies our investigation of algorithmic complexity.

Our algorithm for deciding conjunctive query entailment is given in Table 3 Any occurrence of the word "select" in the description indicates a nondeterministic choice of the algorithm. Step A is a standard preprocessing step for many query answering algorithms. Step B initiates the proof graph and ensures that all nodes are reachable. Variable nodes eventually are reachable through exactly one predecessor node, so the structure of the proof graph resembles the canonical model (compare Property 1 of Section 3). Steps C and D verify that the selected proof graph indeed establishes the existence of the required anonymous elements in the model (C) and the entailment of 
the query's concept atoms (D). At this stage, the proof graph still represents many possible fragments of the canonical model: the edge NFA that connect to variable nodes encode possible generating role paths (in the sense of Property 1 Section 3), each of which leads to a different element in the canonical model. The edges leading to individual nodes have a slightly different meaning: all of the paths they represent must actually exist in any model. Summing up, the proof graph still represents many possible matches between the query and a model of $K B$, though a number of basic decisions on the structure of the considered matches has already been made and it is known that any such match suffices to entail the concept atoms of the query.

Now Step E computes the RBox automata $\mathcal{A}(R)$ of Section 3 and applies a nondeterministic splitting operation, which we define next. We remark that the required "shortest path" exists and is easily found in polynomial time (see [11]).

Definition 3. Consider an NFA $\mathcal{A}=(Q, \Sigma, \delta, i,\{f\})$. A split of $\mathcal{A}$ into $k$ parts is given by NFA $\mathcal{A}_{1}, \ldots, \mathcal{A}_{k}$ with $\mathcal{A}_{j}$ of the form $\left(Q, \Sigma, \delta, q_{j-1},\left\{q_{j}\right\}\right)$ such that $q_{0}=i, q_{k}=f$, and $q_{j} \in Q$ for all $j=1, \ldots, k-1$.

It is easy to see that, if each split automaton $\mathcal{A}_{j}$ accepts some word $w_{j}$, we find that $w_{1} \ldots w_{k}$ is accepted by $\mathcal{A}$. Likewise, any word accepted by $\mathcal{A}$ is also accepted in this sense by some split of $\mathcal{A}$. Since the combination of any split in general accepts less words than $\mathcal{A}$, splitting an NFA usually involves some don't-know nondeterminism.

The intuition underlying this split is that each role NFA $\mathcal{A}(R)$ encodes possible chains of roles that suffice to establish role $R$. Clearly, one such chain must be found for every query atom $R(n, m)$. But the proof graph already imposes a basic structure that defines how elements $n$ and $m$ can be connected, and any match with $R$ must be distributed along the paths of the proof graph. This is implemented by the above split.

Finally, Step F again verifies the earlier choices of the algorithm by comparing the (logically deducible) role chains given by the edge NFA with the role chains that the split NFA require to exist for establishing a match. The case distinction reflects the different intention of edges leading to individual or variable nodes. For edges leading to a variable node, only a single generating role path exists in the canonical model, and all split automata must match one such path (line 27). For edges leading to nominal nodes, all of accepted paths exist in every model. Hence line 24 implements pairwise comparisons of each split NFA with the edge NFA. Concrete implementations for the checks of lines 24 and 27 are discussed in Section 6.

We conclude this section with a small example. Let $K B$ be the knowledge base consisting of the following axioms:

$$
A \sqsubseteq \exists R . B \quad B \sqsubseteq \exists S .\{a\} \quad T \circ R \sqsubseteq T \quad\{a\} \sqsubseteq \exists T .\{b\} \quad\{b\} \sqsubseteq A
$$

with concept names $A$ and $B$, role names $R, S$ and $T$, and individuals $a, b$. Consider the query $\{S(x, y), T(y, x)\}$.

In Step A, the algorithm replaces $y$ by $a$ to obtain the query $\{S(x, a), T(a, x)\}$. The proof graph built in Step B has nodes $N=\{a, b, x\}$ with $L(a)=\{a\}, L(b)=\{b\}$ and $L(x)=B$. Edges are constructed between pairs of elements $(a, b),(b, a),(x, a),(x, b)$, and $(b, x)$ (i.e. $b$ generates $x$ ). The constructed edge NFA are distinguished only by their start and end states (as rule (CR6) of Table 2 is not used), and have the following structure: 


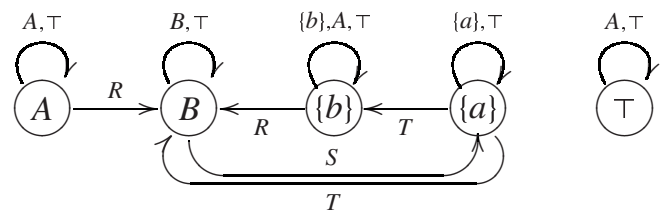

Step C succeeds since every edge automaton accepts some word, and Step D is omitted since no concept atoms appear in the query. The only nontrivial role NFA is $\mathcal{A}(T)$ which accepts any word that starts with $T$ followed by an arbitrary number of $R$. Due to the presence of the query atom $T(a, x)$ this NFA must be split along the path from $a$ over $b$ to $x$, and there is only one split into two NFA that accept nonempty languages. Hence $\mathcal{A}(T(a, x), a, b)$ accepts the single word $T$, and $\mathcal{A}(T(a, x), b, x)$ accepts any sequence of $R$. The only other "split" $\operatorname{NFA} \mathcal{A}(S(x, a), x, a)$ is directly given by $\mathcal{A}(S)$, the NFA accepting only the word $S$. Finally in Step F the three existing split automata are compared to the corresponding edge NFA. $\mathcal{A}(T(a, x), a, b)$ and $E(a, b)$ accept a common word $T, \mathcal{A}(T(a, x), b, x)$ and $E(b, x)$ accept a common word $R$, and $\mathcal{A}(S(x, a), x, a)$ and $E(x, a)$ accept a common word $S$. Hence the query is accepted.

\section{Correctness of the Algorithm}

Proposition 2. Consider a regular consistent $\mathcal{E} \mathcal{L}^{++}$knowledge base KB and a conjunctive query $q$. If the algorithm of Section 4 accepts $q$, then indeed $K B \models q$.

Proof. We use the notation from Section 4 to denote structures computed by the algorithm. When terminating successfully, the algorithm has computed the following:

- A proof graph $(N, L, E)$,

- For each role atom $R(n, m) \in q$, a $k$-split $\mathcal{A}\left(R(n, m), n_{0}, n_{1}\right), \ldots, \mathcal{A}\left(R(n, m), n_{k-1}, n_{k}\right)$ of the NFA $\mathcal{A}(R)$, where $k$ is the length of the shortest path from $n$ to $m$ in $(N, L, E)$.

In the following, let $\mathcal{I}$ be some model of $K B$. To show $K B \models q$, we need to provide a mapping $\pi$ as in Section 2 for $\mathcal{I}$. Since $\mathcal{I}$ is arbitrary, this shows the entailment of $q$. We can derive $\pi$ from the proof graph, and then show its correctness based on the conditions checked by the algorithm.

In Step A, the algorithm replaces variables by individual names or by other variables. This is no problem: whenever a query $q^{\prime}$ is obtained from $q$ by uniformly replacing a variable $x \in \operatorname{Var}(q)$ by an individual $a \in \mathbf{I}$ (or variable $y \in \operatorname{Var}(q)$ ), we have that $K B \models q^{\prime}$ implies $K B \models q$. Indeed, any mapping $\pi^{\prime}$ for $q^{\prime}$ can be extended to a suitable mapping $\pi$ for $q$ by setting $\pi(x):=a^{I}\left(\pi(x):=y^{I}\right)$. Thus we can assume w.l.o.g. that all variables $x \in \operatorname{Var}(q)$ also occur as nodes in the proof graph, i.e. $x \in N$.

In Step F, the algorithm checks non-emptiness of the intersection languages of the NFA $E(n, m)$, and one/all split NFA $\mathcal{A}(F, n, m)$, for each $n, m \in N$ with $E(n, m)$ defined. Thus for any pair $n \in N, m \in \operatorname{Var}(q)$, there is some word $w$ accepted by all of the given NFA. Choose one such word $w(n, m)$. By the definition of the split NFA, $w(n, m)$ is a word over $\mathbf{R}$, and we can assume this to be the case even when no split NFA (but just the single edge automaton) are considered for a given edge. $E(n, m)$ is of the form $\mathcal{A}(L(n), L(m))$ (Definition 2) for the selected labels $L(n)$ and $L(m)$ of the proof graph. 
Now by Theorem 2, the construction of Definition 2, and the fact that $K B$ is consistent, it is easy to see that $E(n, m)$ accepts the word $w(n, m)=R_{1} \ldots R_{l}$ iff $K B \models L(n) \sqsubseteq$ $\exists R_{1} \ldots \exists R_{l} . L(m)$. We employ this fact to inductively construct a mapping $\pi$.

In Step B the algorithm has defined labels $L(x)$ for all $x \in \operatorname{Var}(q)$, and we will retrace this process to construct $\pi$. We claim that the following construction ensures that, whenever a node $n \in N$ is reachable, $\pi(n)$ has been assigned a unique value such that $\pi(n) \in L(n)^{I}$. For starting the induction, set $\pi(a):=a^{I}$ for each $a \in \mathbf{I}$ (which is necessarily reachable and clearly satisfies $\left.\pi(a) \in L(a)^{I}=\{a\}^{I}\right)$. Now assume that in one step the algorithm selected some $x \in \operatorname{Var}(q)$ that was not reachable yet, and node $n \in N$ which is reachable. As noted above, $K B \models L(n) \sqsubseteq \exists R_{1} \ldots \exists R_{l} \cdot L(x)$ where $w(n, x)=$ $R_{1} \ldots R_{l}$, and hence there is an element $e \in L(x)^{I}$ such that $(\pi(n), e) \in R_{1}^{I} \circ \ldots \circ R_{l}^{I}$ (where $\circ$ denotes forward composition of binary relations). Pick one such $e$ and set $\pi(x):=e$. It is easy to see that the claim of the induction is satisfied.

In Step D it has been verified that $L(n) \sqsubseteq C$ holds for each $C(n) \in q$ (using standard polynomial time reasoning for $\mathcal{E} \mathcal{L}^{++}$), so we find $\pi(n) \in C^{\mathcal{I}}$. It remains to show that a similar claim holds for all binary query atoms. Thus consider some role atom $R(n, m) \in q$, and let $n=n_{0}, \ldots, n_{k}=m$ denote the shortest path in the proof graph used to split the role automaton. So far, we have defined $w\left(n_{i}, n_{i+1}\right)$ only for cases where $n_{i+1} \in \operatorname{Var}(q)$. By a slight overloading of notation, we now let $w\left(n_{i}, n_{i+1}\right)$ for $n_{i+1} \in \mathbf{I}$ denote some word accepted by the intersection of $E\left(n_{i}, n_{i+1}\right)$ and the specific split automaton $\mathcal{A}\left(R(n, m), n_{i}, n_{i+1}\right)$, which must exist as the algorithms must have verified nonemptiness of the intersection language. Assuming that $w\left(n_{i}, n_{i+1}\right)=S_{1} \ldots S_{l}$, we note that this still entails $K B \models L\left(n_{1}\right) \sqsubseteq \exists S_{1} \ldots \exists S_{l} . L\left(n_{i+1}\right)$. Since $n_{i+1} \in \mathbf{I}$, this actually shows that $\left(\pi\left(n_{i}\right), \pi\left(n_{i+1}\right)\right) \in S_{1}^{\mathcal{I}} \circ \ldots \circ S_{l}^{\mathcal{I}}$.

The word $w=w\left(n_{0}, n_{1}\right) \ldots w\left(n_{k-1}, n_{k}\right)$ is accepted by $\mathcal{A}(R)$, which is clear from the construction in Definition 3 as the parts $w\left(n_{i}, n_{i+1}\right)$ are accepted by the respective split automata. Assume that $w=R_{1} \ldots R_{k}$. We conclude $(\pi(n), \pi(m)) \in R_{1}^{I} \circ \ldots \circ R_{k}^{I}$ from the construction of $\pi$ and the above observations for the case of edges connecting to individual elements. Thus by Proposition 1 we have $(\pi(n), \pi(m)) \in R^{I}$ as required.

It remains to show that the algorithm is also complete. This is done by demonstrating that there are suitable nondeterministic choices that enable the algorithm to accept a query whenever it is entailed. To guide those choices, we use the canonical model $I$ introduced in Section 3 .

Proposition 3. Consider a regular consistent $\mathcal{E} \mathcal{L}^{++}$knowledge base $K B$ and a conjunctive query q. If $K B \models q$, then there is a sequence of nondeterministic choices for the algorithm of Section 4 such that it accepts $q$.

Proof. Consider the canonical model $\mathcal{I}$ as constructed above. Since $K B=q$ and $\mathcal{I}=$ $K B$, there is some mapping $\pi$ such that $\mathcal{I}, \pi \models q$. We will use $\pi$ to guide the algorithm.

In Step A, a variable $x \in \operatorname{Var}(q)$ is replaced by $n \in \operatorname{Var}(q) \cup \mathbf{I}$ whenever $\pi(x)=\pi(n)$. For Step B, we choose the labelling $L$ of the proof graph by setting $L(e):=\iota(\pi(e))$. As argued in the proof of Lemma $1, \delta \in C^{\mathcal{I}}$ iff $K B \models \iota(\delta) \sqsubseteq C$, and hence we conclude that $\pi(e) \in C^{\mathcal{I}}$ implies that $K B \models L(e) \sqsubseteq C$ for all $e \in \mathbf{I} \cup \operatorname{Var}(q)$. Thus all unary atoms of $q$ are accepted by the algorithm. 
Now in each step of the generation of the edges $E$ of the proof graph, the algorithm needs to pick some (unreachable) $x \in \operatorname{Var}(q)$ and some reachable node $n$. We will utilise the properties established in Section 3. By Property 1, there is a unique generating chain for each $\pi(x)$ where $x$ is not reachable within the proof graph yet. Moreover, since the chain of Property 1 is unique and shortest, it is also acyclic. Hence there is some unreachable $x$ such that $\pi(x)$ is not generated by any element of the form $\pi(y)$ with $y$ unreachable. Pick one such element $x$. Finally select one element $n \in \mathbf{I} \cup \operatorname{Var}(q)$ such that $\pi(n)$ generates $\pi(x)$, and such that there is no element $m$ for which $\pi(m)$ generates $\pi(x)$ and $\pi(n)$ generates $\pi(m)$. Construct an edge $E(m, x)$.

Now for any elements $n$ and $m$ of the query, with $m \in \operatorname{Var}(q)$ and $E(n, m)$ defined, the automaton $E(n, m)$ accepts a non-empty language. This is seen by combining Property 2 with Theorem 2, where the second case of the theorem is excluded since $K B$ is consistent. The algorithm's checks in Step C thus succeed.

The algorithm now has completed the proof graph construction, and the selection of split automata is required next. For all query atoms $R(n, m)$, we find that $(\pi(n), \pi(m)) \in$ $R^{I}$, and thus we can apply Property 3 to obtain a respective chain of elements and role names, which we denote as $\delta_{0} \ldots \delta_{k}$ and $R_{0} \ldots R_{k-1}$ in the remainder of this proof.

Let $j>0$ denote the largest index of $\delta_{0} \ldots \delta_{k}$, such that $\delta_{j}$ is of the form $\pi\left(e_{1}\right)$ for some $e_{1} \in \mathbf{I}$, if any such element exists. Otherwise, let $j>0$ denote the smallest index such that $\delta_{i}$ is of the form $\pi\left(e_{1}\right)$ for any $e_{1} \in \operatorname{Var}(q)$. We claim that there is a connection between $n$ and $e_{1}$ in the proof graph. Clearly, this is true if $e_{1} \in \mathbf{I}$ since these edges were constructed explicitly. Otherwise, Property 1 and our choice of $e_{1}$ imply that an edge from $n$ to $e_{1}$ was constructed by the algorithm. Starting by $\delta_{j+1}$, find all elements $\delta_{i}$ of the form $\pi(e), e \in \operatorname{Var}(q)$, and label them consecutively as $e_{2}, \ldots, e_{l}$. Note that this sequence can be empty, in which case we define $l:=1$. Obviously, $e_{l}=m$. We claim that $n=e_{0} \ldots e_{l}=m$ is the shortest path from $n$ to $m$ within the proof graph. We already showed the connection between $n=e_{0}$ and $e_{1}$. The connections between $e_{i}$ and $e_{i+1}$ are also obvious, since each $e_{1}$ generates $e_{i+1}$ by definition. Since the latter path is also the only path from $e_{1}$ to $e_{l}$, the overall path is clearly the shortest connection.

The algorithm now splits $\mathcal{A}(R)$ along the path $n=e_{0} \ldots e_{l}=m$. For each $e_{i}$, there is an index $j(i)$ such that $\delta_{j(i)}=\pi\left(e_{i}\right)$. Hence, for each pair $\left(e_{i}, e_{i+1}\right)$, there is a corresponding sequence of roles $R_{j(i)+1} \ldots R_{j(i+1)}$ which we denote by $r_{i}(i=0, \ldots, l-1)$, and the concatenation of those sequences yields the original $R_{0} \ldots R_{k-1}$. By Proposition 1 and Property 3 , the automaton $\mathcal{A}(R)$ accepts the word $R_{0} \ldots R_{k-1}$. To split the automaton, we consider one accepting run and define $q_{i}$ to be the state of the automaton after reading the partial sequence $r_{i}$, for each $i=0, \ldots, l-1$. The states $q_{i}$ are now used to construct the split automata $\mathcal{A}_{i}$, and it is easy to see that those automata accept the sequences $r_{i}$.

Now assume that all required split automata have been constructed in this way. Consider any pair of query elements $e, e^{\prime} \in \mathbf{I} \cup \operatorname{Var}(q)$ for which a split automaton $\mathcal{A}\left(F, e, e^{\prime}\right)$ was constructed using a partial sequence of roles $r$. We claim that the edge automaton $E\left(e, e^{\prime}\right)$ accepts $r$. Indeed, this follows from Property 2 and Theorem 2 This shows non-emptiness of intersections between any single split automaton and the corresponding edge automaton in the proof graph, and thus suffices for the case where $e^{\prime} \in \mathbf{I}$.

Finally, consider the case that $e^{\prime} \in \operatorname{Var}(q)$, and assume that two split automata $\mathcal{A}\left(F, e, e^{\prime}\right)$ and $\mathcal{A}\left(F^{\prime}, e, e^{\prime}\right)$ have been constructed for the given pair, based on two 
partial role sequences $r$ and $r^{\prime}$. We claim that $r=r^{\prime}$. Indeed, this is obvious from the fact that $r$ and $r^{\prime}$ both correspond to the unique generating sequence of roles for the elements $e$ and $e^{\prime}$, which is part of the sequence constructed for Property 1 . This shows that $r$ is accepted both by $\mathcal{A}\left(F, e, e^{\prime}\right)$ and by $\mathcal{A}\left(F^{\prime}, e, e^{\prime}\right)$. We conclude that the intersection of all split automata and the edge automaton $E\left(e, e^{\prime}\right)$ is again non-empty.

The algorithm thus has completed all checks successfully and accepts the query.

\section{Complexity of Query Answering for $\mathcal{E} \mathcal{L}^{++}$}

Finally, we harvest a number of complexity results from the algorithm of Section 4.

Lemma 2. Given a regular $\mathcal{E} \mathcal{L}^{++}$knowledge base $K B$ and a conjunctive query $q$, the entailment problem $K B \models q$ is hard for $\mathrm{NP}$ w.r.t. the size of $q$, hard for $\mathrm{P}$ w.r.t. the size of the ABox of $K B$, and hard for PSPACE w.r.t. to the combined problem size, even when restricting to simple RBoxes.

The hardness proofs in [11] apply known hardness results for the data-complexity of instance checking in fragments of $\mathcal{E} \mathcal{L}$ [12], evaluation of single Datalog clauses (NPcomplete, [13]), and emptiness of NFA intersection languages (PSPACE-complete, [14]).

We remark that the above results are quite generic, and can be established for many other DLs. Especially, NP-hardness w.r.t. knowledge base size can be shown for any logic that admits an ABox, whereas PSPACE hardness of the combined problem follows whenever the DL additionally admits role composition and existential role restrictions.

Lemma 3. Given a regular $\mathcal{E} \mathcal{L}^{++}$knowledge base $K B$ and a conjunctive query $q$, the entailment problem $K B \models q$ can be decided in $\mathrm{P}$ w.r.t. the size of the knowledge base, in NP w.r.t. the size of the query, and in PSPACE w.r.t. the combined problem size, given that RBoxes are simple whenever KB is not fixed.

Proof. First consider Step A of Table 3. It clearly can be performed nondeterministically in polynomial time. If the query is fixed, the number of choices is polynomially bounded, and so the whole step is executable in polynomial time.

Similar observations hold for Step B. Concept names and automata for edges can be assigned in polynomial time by a nondeterministic algorithm (and thus in polynomial space). If the query has fixed size, available choices again are polynomial in the size of $K B$ : the assignment of labels $L$ admits at most $|\mathbf{C}|^{|\operatorname{Var}(q)|}$ different choices, and for each such choice, there are at most $n^{2}$ possible proof graphs, where $n$ is the number of nodes in the graph. Since $n$ and $|\operatorname{Var}(q)|$ are considered fixed, this yields a polynomial bound.

Further nondeterminism occurs in Step E. But if the query is fixed, each of the polynomially many proof graphs dictates a number of splits that is bounded by the size of the query $m$. Since splitting a role NFA into $k$ parts corresponds to selecting $k$ (not necessarily distinct) states from this NFA, there are $\left|Q_{\mathcal{A}}\right|^{k}$ different ways of splitting $\mathcal{A}$. Since $k$ is bounded by the size of the query $m$, we obtain an upper bound $|Q|^{m^{m}}$ that is still polynomial in the size of $K B$ (which, by our assumptions on simplicity of the RBox, determines the maximum number of states $|Q|$ of some role NFA). If the query is not fixed, splitting can be done nondeterministically in polynomial time. 
Now for Step F, the algorithm essentially has to check the emptiness of intersection languages of various automata. Given NFA $\mathcal{A}_{1}, \ldots, \mathcal{A}_{l}$, this check can be done in two ways, each being worst-case optimal for different side conditions of the algorithm:

(1) Initialise state variables $q_{1}, \ldots, q_{l}$ as being the initial states of the involved NFA. Then nondeterministically select one input symbol and one transition for this symbol in each of the considered NFA, and update the states $q_{j}$ accordingly. The algorithm is successful if at some stage each $q_{j}$ is a final state of the automaton $\mathcal{A}_{j}$. The algorithm runs in NPSPACE w.r.t. the accumulated size of the input automata.

(2) Iteratively compute the intersection NFA for $\mathcal{A}_{j}=\left(Q_{j}, \Sigma, \delta_{j}, i_{j}, F_{j}\right)$ and $\mathcal{A}_{j+1}=$ $\left(Q_{j+1}, \Sigma, \delta_{j+1}, i_{j+1}, F_{j+1}\right)$. This intersection is the NFA $\left(Q_{j} \times Q_{j+1}, \Sigma, \delta,\left(i_{j}, i_{j+1}\right), F_{j} \times\right.$ $\left.F_{j+1}\right)$, with $\delta\left(\left(a_{1}, b_{1}\right),\left(a_{2}, b_{2}\right)\right)=\delta\left(a_{1}, a_{2}\right) \cap \delta\left(b_{1}, b_{2}\right)$. The algorithm is successful if the intersection is non-empty. This construction is polynomial if the number of the input NFA is known to be bounded.

Method (1) establishes a general (nondeterministic) polynomial space procedure, which by Savitch's Theorem is also in PSPACE. Method (2) can be used to establish tighter bounds in special cases: each intersection might cause a quadratic increase of the size of the NFA, but the number of required intersections is bounded if $K B$ or $q$ are fixed. Indeed, if the query is fixed, the number of required intersections is bounded by the overall number of role atoms in the query. If the knowledge base is fixed, the number of interesting intersections is bounded by the number of split NFA that can be produced from role NFA constructed from the RBox, which is bounded by a fixed value. In both cases, checking intersections can be done deterministically in polynomial time.

The below table summarises some common complexity measures for the case of conjunctive query answering in regular $\mathcal{E} \mathcal{L}^{++}$knowledge bases. Whenever the RBox is variable, we assume that it is simple. It should be remarked that TBox and ABox could always be considered variable without increasing any of the given complexities.

\begin{tabular}{|c|c|c|c|c|c|}
\hline & \multicolumn{4}{|c|}{ Variable parts: } & \\
& Query & RBox & TBox & ABox & Complexity \\
\hline \hline Combined complexity & $\times$ & $\times$ & $\times$ & $\times$ & PSPACE-complete \\
\hline Query complexity & $\times$ & & & & NP-complete \\
\hline Schema complexity & & $\times$ & $\times$ & $\times$ & P-complete \\
\hline Data complexity & & & & $\times$ & P-complete \\
\hline
\end{tabular}

\section{Conclusion}

We have proposed a novel algorithm for answering conjunctive queries in $\mathcal{E} \mathcal{L}^{++}$knowledge bases, which is worst-case optimal under various assumptions. To the best of our knowledge, this also constitutes the first inference procedure for conjunctive queries in a DL that supports complex role inclusions (including composition of roles) in the sense of OWL 1.1. Showing undecidability of conjunctive queries for unrestricted $\mathcal{E} \mathcal{L}^{++}$, we illustrated that the combination of role atoms in queries and complex role inclusion axioms can indeed make reasoning significantly more difficult. 
A compact automata-based representation of role chains and (parts of) models allowed us to establish polynomial bounds for inferencing in various cases, thus identifying querying scenarios that are still tractable for $\mathcal{E} \mathcal{L}^{++}$. Conjunctive queries inherently introduce some nondeterministism, but automata can conveniently represent sets of possible solutions instead of considering each of them separately. We therefore believe that the presented algorithm can be a basis for actual implementations that introduce additional heuristics to ameliorate nondeterminism.

\section{References}

1. Chandra, A.K., Merlin, P.M.: Optimal implementation of conjunctive queries in relational data bases. In: Hopcroft, J.E., Friedman, E.P., Harrison, M.A. (eds.) STOC 1977. Proc. 9th annual ACM Symposium on Theory of Computing, pp. 77-90. ACM Press, New York (1977)

2. Horrocks, I., Sattler, U., Tessaris, S., Tobies, S.: How to decide query containment under constraints using a description logic. In: Parigot, M., Voronkov, A. (eds.) LPAR 2000. LNCS (LNAI), vol. 1955, pp. 326-343. Springer, Heidelberg (2000)

3. Hustadt, U., Motik, B., Sattler, U.: A decomposition rule for decision procedures by resolution-based calculi. In: Baader, F., Voronkov, A. (eds.) LPAR 2004. LNCS (LNAI), vol. 3452, pp. 21-35. Springer, Heidelberg (2005)

4. Ortiz, M.M., Calvanese, D., Eiter, T.: Data complexity of answering unions of conjunctive queries in SHIQ. In: Proc. 2006 Description LogicWorkshop (DL 2006), CEUR Electronic Workshop Proceedings (2006), http: //ceur-ws.org/

5. Ortiz, M.M., Calvanese, D., Eiter, T.: Characterizing data complexity for conjunctive query answering in expressive description logics. In: AAAI 2006. Proc. 21st Nat. Conf. on Artificial Intelligence (2006)

6. Glimm, B., Horrocks, I., Lutz, C., Sattler, U.: Conjunctive query answering for the description logic SHIQ. In: IJCAI 2007. Proc. 21st Int. Joint Conf. on Artificial Intelligence, Hyderabad, India (2007), available at http://wWw .ijcai.org/papers07/contents.php

7. Baader, F., Brandt, S., Lutz, C.: Pushing the EL envelope. In: IJCAI 2005. Proc. 19th Int. Joint Conf. on Artificial Intelligence, Morgan-Kaufmann Publishers, Edinburgh, UK (2005)

8. Krisnadhi, A., Lutz, C.: Data complexity in the EL family of DLs. In: Proc. DL 2007. CEUR Electronic Workshop Proceedings (2007)

9. Horrocks, I., Sattler, U.: Decidability of SHIQ with complex role inclusion axioms. In: Gottlob, G., Walsh, T. (eds.) IJCAI 2003. Proc. 18th Int. Joint Conf. on Artificial Intelligence, Acapulco, Mexico, pp. 343-348. Morgan-Kaufmann Publishers, San Francisco (2003)

10. Horrocks, I., Kutz, O., Sattler, U.: The even more irresistible SROIQ. In: KR 2006. Proc. 10th Int. Conf. on Principles of Knowledge Representation and Reasoning, pp. 57-67. AAAI Press, Stanford, California, USA (2006)

11. 11. Krötzsch, M., Rudolph, S.: Conjunctive queries for EL with role composition. Technical report, Universität Karlsruhe (TH), Germany (2007), available at http://www.aifb.uni-karlsruhe.de/Publikationen/showPublikation? publ_id=1463

12. Calvanese, D., De Giacomo, G., Lembo, D., Lenzerini, M., Rosati, R.: Data complexity of query answering in description logics. In: KR 2006. Proc. 10th Int. Conf. on Principles of Knowledge Representation and Reasoning, pp. 260-270 (2006)

13. Dantsin, E., Eiter, T., Gottlob, G., Voronkov, A.: Complexity and expressive power of logic programming. ACM Computing Surveys 33, 374-425 (2001)

14. Kozen, D.: Lower bounds for natural proof systems. In: Proc. 18th Symp. on the Foundations of Computer Science, pp. 254-266 (1977) 